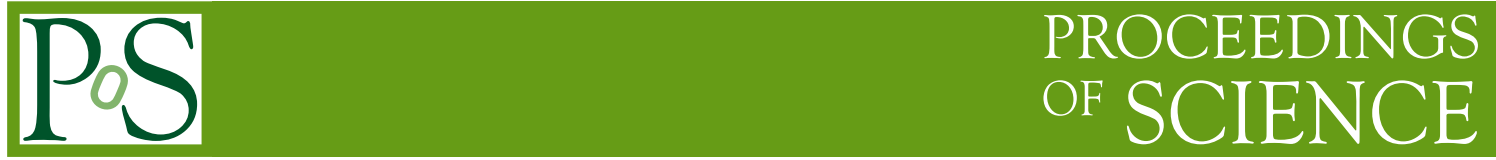

\title{
Deciphering the Extreme Universe
}

\author{
Angela V. Olinto* \\ Department of Astronomy \& Astrophysics, EFI, KICP, \\ The University of Chicago, \\ 5640 S. Ellis Ave, Chicago, IL 60637, USA Chicago \\ E-mail: olinto@oddjob.uchicago.edu
}

The current state and future prospects of ultra high energy cosmic ray physics are reviewed.

These cosmic rays with energies well above $10^{18} \mathrm{eV}$ are messengers of an unknown extremely

high-energy universe.

28th Johns Hopkins Workshop on Current Problems in Particle Theory

June 5-8, 2004

Johns Hopkins University Homewood campus - Bloomberg Center for Physics and Astronomy, Baltimore, Maryland

* Speaker. 


\section{Introduction}

Cosmic rays have been known to be of cosmic origin since 1912 when Victor Hess took electroscopes in balloons above $5000 \mathrm{~m}$. By 1938 Pierre Auger had shown that cosmic ray primaries reach energies in excess of $10^{15} \mathrm{eV}$ with the detection of extensive air-showers [1].Since then cosmic rays have been observed up to $\sim 10^{20} \mathrm{eV}$. Fermi acceleration in supernova remnants may be responsible for accelerating cosmic rays below $\sim 10^{15} \mathrm{eV}$, but more powerful sources seem to be required for the higher energy events. No sources of cosmic rays have been identified and their origin remains a mystery about to become a century old.

Figure 1 shows a compilation of direct and indirect (via air showers) cosmic ray observations unified into a single spectrum. The spectrum is well fit by power-laws with spectral index $\gamma \simeq 2.7$ for energies below $\sim 10^{15} \mathrm{eV}$ and $\gamma \simeq 3$ for energies above $\sim 10^{15} \mathrm{eV}$, with a varying low energy cutoff due to solar magnetic fields. The composition of cosmic rays is well understood below $\sim 10^{14} \mathrm{eV}$. The spectrum is dominated by protons, followed by He, C, N, O, and finally Fe nuclei. At higher energies, indirect evidence points to a change from proton to $\mathrm{Fe}$ dominated spectrum between $\sim 10^{15} \mathrm{eV}$ and $\sim 10^{17} \mathrm{eV}$ [2] with a possible change back to protons above $\sim 10^{18} \mathrm{eV}$ [3] as shown in Figure 2. For energies above $\sim 10^{19} \mathrm{eV}$ the composition is unknown.

At the highest energies, the present state of observations is particularly puzzling. Fortunately, the necessary experiments to resolve these puzzles are starting operate now. The ultra-high energy cosmic ray (UHECR) puzzles begin with the lack of the predicted Greisen-Zatsepin-Kuzmin (GZK) cutoff [4]. Contrary to earlier expectations, cosmic rays with energies above $10^{20} \mathrm{eV}$ have been detected by a number of experiments (for reviews see [5, 6]). If these particles are protons, they are likely to originate in extragalactic sources, since at these high energies the Galactic magnetic field cannot confine protons in the Galaxy. However, extragalactic protons with energies above a few times $\sim 10^{20} \mathrm{eV}$ produce pions through interactions with the cosmic microwave background (CMB) and consequently lose significant amounts of energy as they traverse intergalactic distances. Thus, in addition to the extraordinary energy requirements for astrophysical sources to accelerate protons to $\gtrsim 10^{20} \mathrm{eV}$, the photopion threshold reaction suppresses the observable flux above $\sim 10^{20} \mathrm{eV}$. These conditions were expected to cause a natural high-energy limit to the cosmic ray spectrum known as the GZK cutoff [4].

As reported by the most recent compilation of the Akeno Giant Airshower Array (AGASA) data [7], the spectrum of cosmic rays does not end at the expected GZK cutoff. The significant flux observed above $10^{20} \mathrm{eV}$ together with a nearly isotropic distribution of event arrival directions challenges astrophysically based explanations as well as new physics alternatives. In addition, the reported small scale clustering [8] tends to rule out most scenarios.

This challenging state of affairs is stimulating both for theoretical investigations as well as experimental efforts. The explanation may hide in the experimental arena such as an over estimate of the flux at the highest energies. This explanation has been proposed by the High Resolution Fly's Eye (HiRes) collaboration based on an analysis of their monocular data [9] which is consistent with a GZK feature. Currently, these two experiments with the largest exposures have conflicting results at the highest energies (above $\sim 10^{20} \mathrm{eV}$ ) where limited statistics and systematic errors prevent a clear resolution. As new experiments come on line, the structure of the GZK feature will become clear. In any scenario (GZK feature or not), events past $10^{20} \mathrm{eV}$ pose theoretical challenges which 


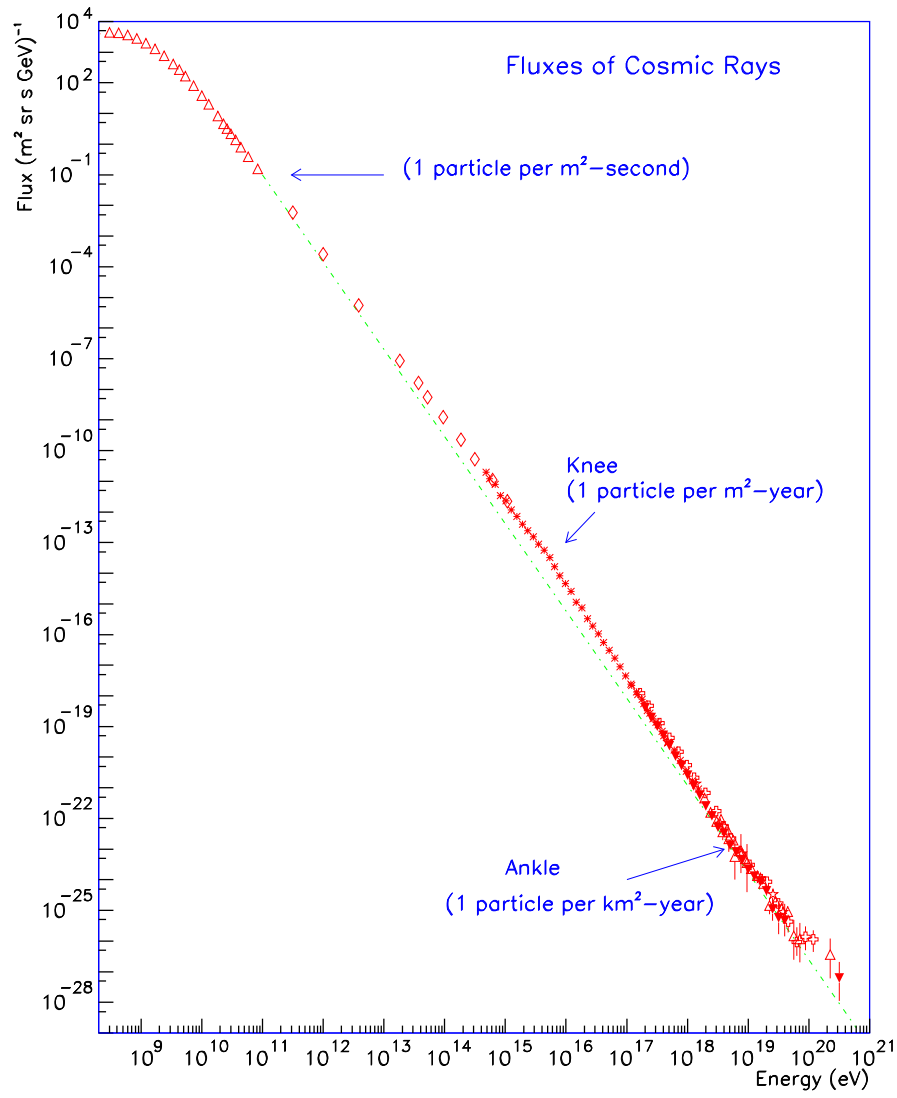

Figure 1: Spectrum of cosmic rays.

will be explained in the future by either astrophysically novel sources or new fundamental physics.

\section{Present State of UHECR observations}

Ultra-high energy cosmic rays are the highest energy messengers of the present universe. The highest energy cosmic photons observed thus far reach up to $\sim 10^{13} \mathrm{eV}$. Extragalactic photons of higher energies loose a significant fraction of their energies due to pair production in the cosmic background radiation as they traverse large regions of intergalactic space. In contrast, cosmic rays are observed with energies as high as $3 \times 10^{20} \mathrm{eV}$ and with fluxes well above upper limits on high-energy gamma-ray fluxes.

However, the origin of cosmic rays remains a mystery hidden by the fact that these relativistic particles do not point back to their sources. These charged particles are deflected by magnetic fields that permeate interstellar and intergalactic space. Galactic magnetic fields are known to be around a few micro Gauss in the Galactic disk and are expected to decay exponentially away from the disk [10]. Intergalactic fields are observed in dense clusters of galaxies, but it is not clear if there are intergalactic magnetic fields in the Local Group or the Local Supergalactic Plane. On larger scales, magnetic fields are known to be weaker than $\sim 10$ nano Gauss [11].

As cosmic ray energies reach $10^{20} \mathrm{eV}$ per charged nucleon, Galactic and intergalactic magnetic fields cannot bend particle orbits significantly and pointing to cosmic ray sources becomes feasible. 


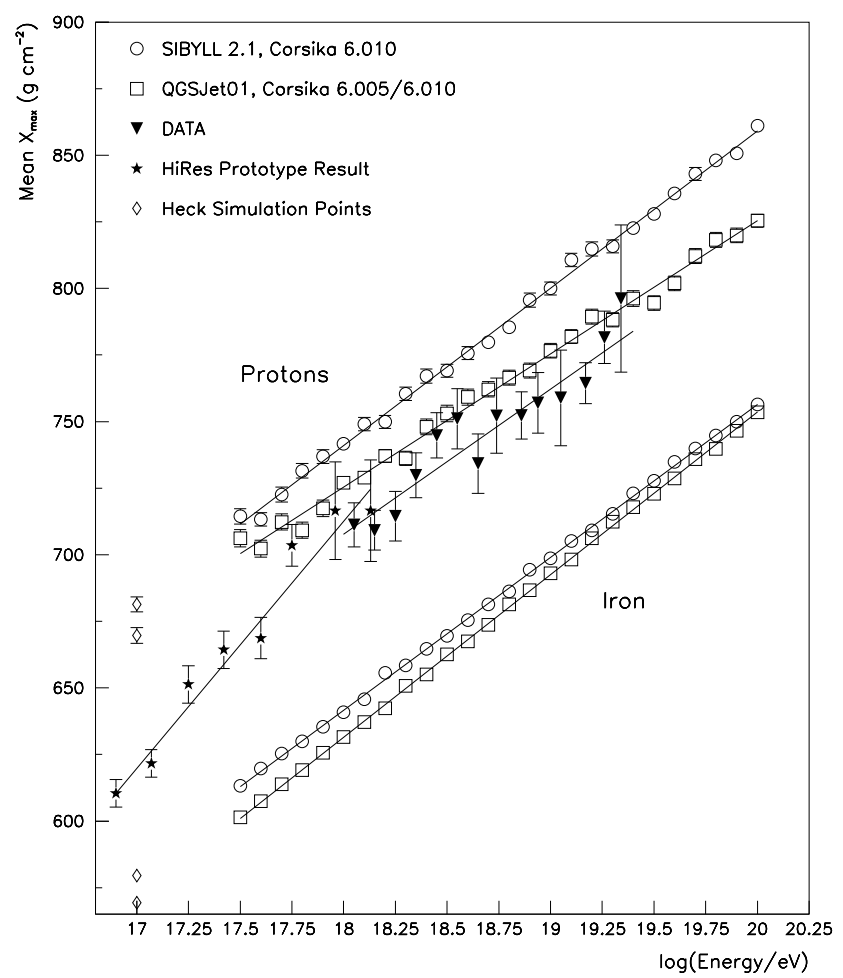

Figure 2: Composition of UHECRs as reported by HiRes. Elongation rate versus energy compared to predictions for QGSJet and SIBYLL protons and iron.

Recent high-resolution simulations of large-scale structure formation in a $\Lambda$ CDM universe can follow the magnetic field evolution from seed fields to present fields in galaxies and clusters [12]. The intergalactic medium fields in these simulations are consistent with Faraday rotation measurements at the $10^{-9}-10^{-8}$ Gauss level. In addition to simulating the field evolution, cosmic ray protons are propagated through a volume of $110 \mathrm{Mpc}$ radius. Figure 3 shows that the deflection from the source position to the arrival direction for protons with arrival energy of $4 \times 10^{19} \mathrm{eV}$ can reach around 1 degree in the densest regions [12]. For protons arriving with $10^{20} \mathrm{eV}$ the deflections are less than $\sim 0.1^{\circ}$ (which is significantly smaller than the resolution of UHECR observatories) [12]. Therefore, at ultra high energies there is finally the opportunity to begin cosmic ray astronomy.

In addition to the ability to point back to the source position, cosmic ray protons of energies around $10^{20} \mathrm{eV}$ should display a well-known spectral feature called the GZK cutoff [4]. This cutoff was proposed in 1966 by Greisen, Zatsepin and Kuzmin as a natural end to the cosmic ray spectrum due to photopion production off the then recently discovered cosmic microwave background radiation. The presence of microwave photons through cosmic space induces the formation and subsequent decay of the $\Delta^{+}$resonance for protons with energies above $\sim 10^{20} \mathrm{eV}$ that traverse distances longer than $\sim 50 \mathrm{Mpc}$. The effect of photopion production is to decrease the energy of protons from distant sources resulting in a hardening of the spectrum between $10^{19} \mathrm{eV}$ and $10^{20}$ $\mathrm{eV}$ followed by a sharp softening past $10^{20} \mathrm{eV}$. Depending on the maximum energy of ultra high- 


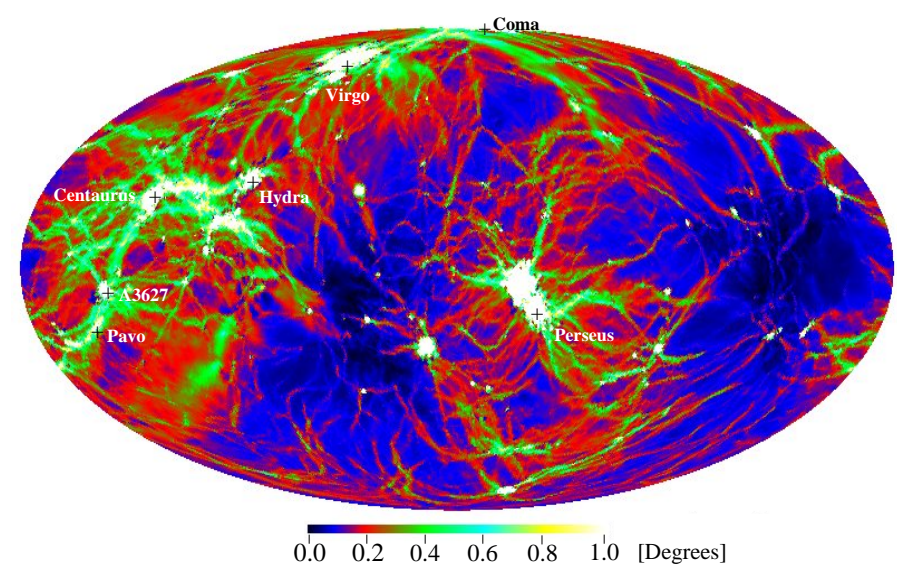

Figure 3: Shown is a full sky map of expected angular deflections for extragalactic cosmic ray sources using simulations of large scale structure formation with magnetic fields. Cosmic ray protons with arrival energy $E=4 \times 10^{19} \mathrm{eV}$ were propagated through the whole simulation volume within a radius of $110 \mathrm{Mpc}$ around the position of the Galaxy. The coordinate system is galactic, with the galactic anti-center in the middle of the map.

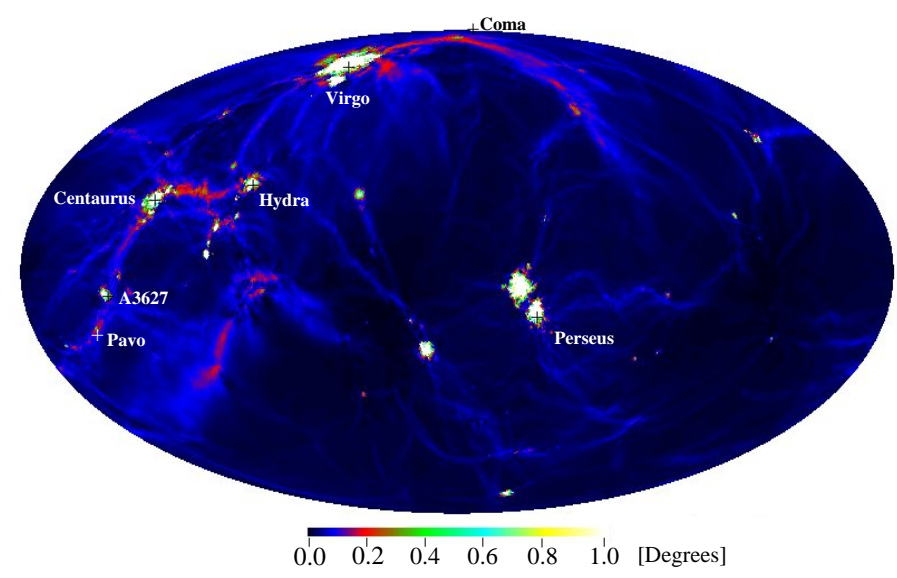

Figure 4: Shown is a map as in previous figure of the expected angular deflections for protons with arrival energy $E=1 \times 10^{20} \mathrm{eV}$ including energy losses in the propagation.

energy cosmic ray sources and their distribution in the universe, the spectrum may harden again past the GZK feature displaying the injected spectrum of nearby sources.

The search for the origin of the highest energy particles is being undertaken by a number of experiments. At present, observations of cosmic rays at the highest energies have yielded measurements of the spectrum, arrival direction distribution, and composition of UHECRs below $10^{20} \mathrm{eV}$. The cosmic ray spectrum past $10^{20} \mathrm{eV}$ should show the presence or absence of the GZK feature, which can be related to the type of primary (e.g., protons) and source (injection spectrum and spatial distribution) of UHECRs. Currently, the two largest exposure experiments, the Akeno Giant Airshower Array (AGASA) and the High Resolution Fly's Eye (HiRes) have conflicting results at the highest energies (above $\sim 10^{20} \mathrm{eV}$ ) where limited statistics and systematic errors prevent a clear resolution. 


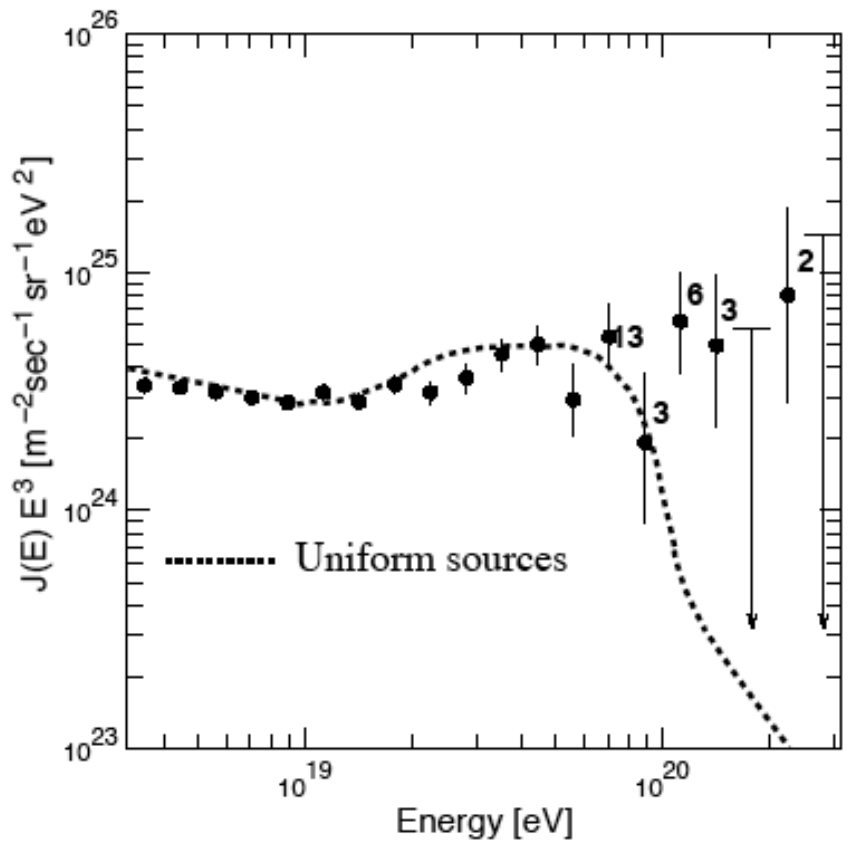

Figure 5: AGASA spectrum.

AGASA is a $100 \mathrm{~km}^{2}$ ground array of scintillator and muon detectors. AGASA data shows a distribution of arrival directions which is mainly isotropic with an indication of clustering of cosmic rays at the highest energies and smallest angles [8]. In addition, the spectrum shows the lack of a GZK cutoff around $10^{20} \mathrm{eV}$ (see figure 5). The flux above $10^{20} \mathrm{eV}$ does not show the expected GZK cutoff with the detection of 11 Super-GZK events, i.e., 11 events with energies above $10^{20}$ $\mathrm{eV}$ [7]. These findings argue against the notion of extragalalactic proton sources of UHECRs and for a unexpected new source at the highest energies.

In contrast, the HiRes monocular spectrum indicates smaller fluxes past $10^{20} \mathrm{eV}$ which is consistent with a GZK feature [9]. HiRes reports only two events with energies above $10^{20} \mathrm{eV}$. HiRes is composed of fluorescence telescopes built in two different sites in the Utah desert to be used as a stereo fluorescence detector. While stereo results do not have comparable exposure to AGASA yet, monocular data do have comparable exposure. Mono HiRes analysis shows no evidence of clustering of arrival directions on small scales [13] and a decrease in flux consistent with the GZK feature. In addition to the spectrum and distribution of arrival directions, HiRes data indicates that between $10^{18} \mathrm{eV}$ and $10^{19.3} \mathrm{eV}$ the composition shifts from a heavier (iron dominated) component to lighter (proton dominated) component.

The implications of the differing results from AGASA and HiRes are especially intriguing at the highest energies. The discrepancies between HiRes and AGASA spectra corresponds to 30\% systematic error in energy scales. Possible sources of systematic errors in the energy measurement of the AGASA experiment were comprehensively studied to be at around $18 \%$ [14]. Systematic errors in HiRes are still being evaluated, but are likely to be dominated by uncertainties in the absolute fluorescence yield, the atmospheric corrections, and the calibration of the full detector, which could amount to at least $\sim 20 \%$ systematic errors in energy calibration. 


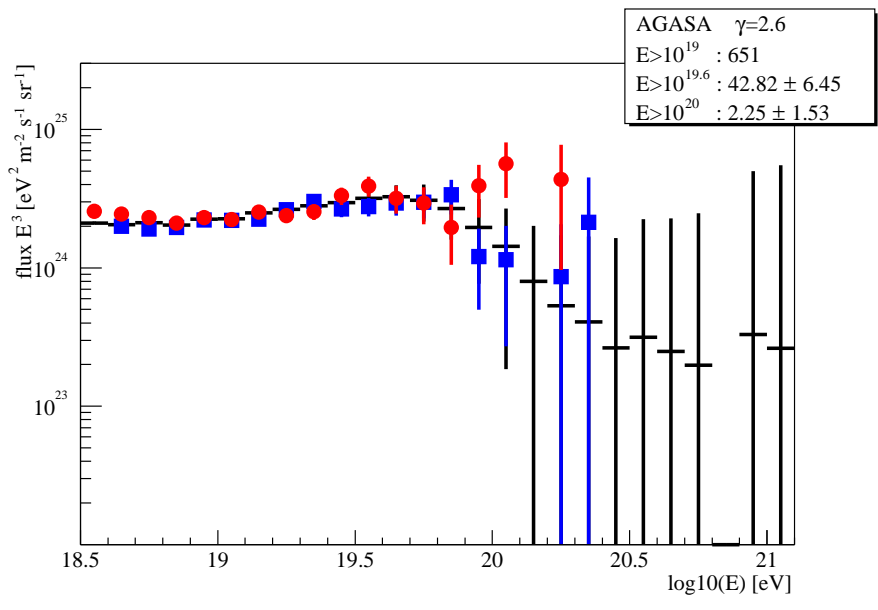

Figure 6: AGASA with $-15 \%$ energy shift and HiRes with $+15 \%$ shift.

Although control of systematic errors is crucial, the statistics accumulated by both HiRes and AGASA are not large enough for a clear measurement of the GZK feature. Figs. 6 shows the range of 400 simulated spectra of protons propagating in intergalactic space with injection spectral index of 2.8 for AGASA and 2.6 for HiRes exposures. In addition, the data from AGASA and HiRes are shown with a systematic energy shift of $-15 \%$ for AGASA and $+15 \%$ for HiRes [15]. The disagreement between the two experiments is only about $2 \sigma$ using these arbitrarily chosen systematic corrections, which are well within the possible range of systematic errors.

The systematic energy shifts between AGASA and HiRes through the range of observed energies is more easily seen when the two spectra are plotted on a flux versus energy plot (see Figure 7). In addition, the discrepancies between the two experiments are not as accentuated as in the traditional plots of flux times $E^{3}$. Finally, the low exposure above $10^{20} \mathrm{eV}$ of both experiments prevents an accurate determination of the GZK feature or lack of it. The lessons for the future are clear: improve the statistics significantly above $10^{20} \mathrm{eV}$ and understand the sources of systematic errors.

\section{Possible Sources of UHECRs}

The puzzle presented by the observations of cosmic rays above $10^{20} \mathrm{eV}$ have generated a number of proposals that can be divided into Astrophysical Zevatrons and New Physics models. Astrophysical Zevatrons are also referred to as bottom-up models and involve searching for acceleration sites in known astrophysical objects that can reach $\mathrm{ZeV}$ energies. New Physics proposals can be either hybrid or pure top-down models. First we discuss astrophysical Zevatrons in this section followed by new physics models.

\subsection{Astrophysical Zevatrons}

Cosmic rays can be accelerated in astrophysical plasmas when large-scale macroscopic motions, such as shocks, winds, and turbulent flows, are transferred to individual particles. The maximum energy of accelerated particles, $E_{\max }$, can be estimated by requiring that the gyroradius of 


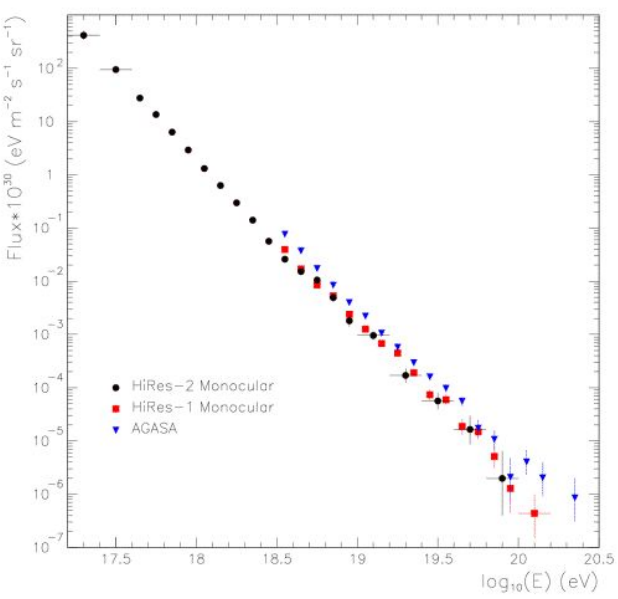

Figure 7: AGASA and HiRes flux versus energy.

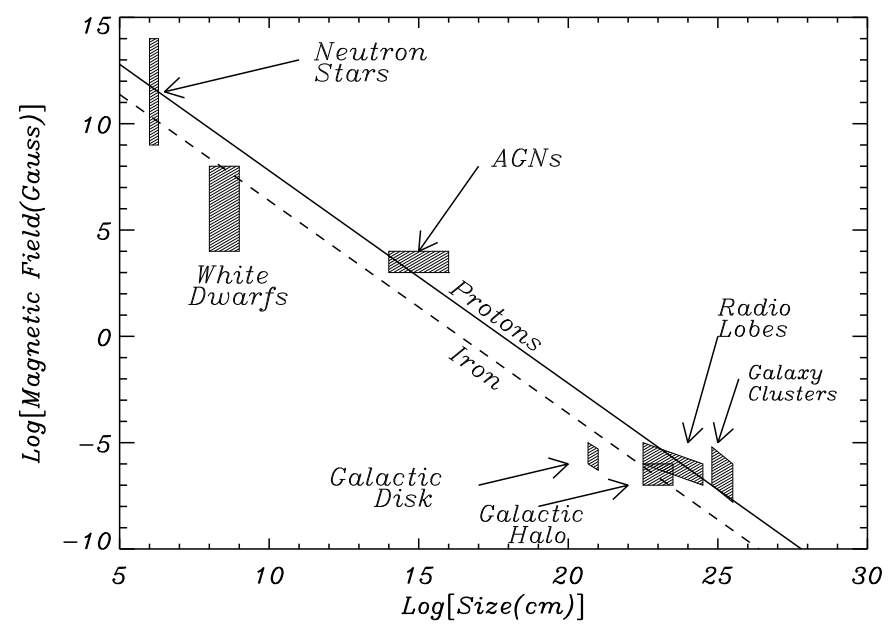

Figure 8: $B$ vs. $L$, for $E_{\max }=10^{20} \mathrm{eV}, Z=1$ (dashed line) and $Z=26$ (solid line).

the particle be contained in the acceleration region: $E_{\max }=Z e B L$, where $Z e$ is the charge of the particle, $B$ is the strength and $L$ the coherence length of the magnetic field embedded in the plasma. For $E_{\max } \gtrsim 10^{20} \mathrm{eV}$ and $Z \sim 1$, the only known astrophysical sources with reasonable $B L$ products are neutron stars, active galactic nuclei (AGNs), radio lobes of AGNs, and clusters of galaxies. Figure 8 (know as a Hillas plot [17]) highlights the $B$ vs. $L$ for these objects.

Although very large, shocks in clusters of galaxies are not able to accelerate protons to energies above $\sim 10^{19} \mathrm{eV}$ [18]. In addition, the propagation in clusters and in the extragalactic medium should generate a GZK feature. Jets from the central black-hole of an active galaxy end at a termination shock where the interaction of the jet with the intergalactic medium forms radio lobes and 'hot spots'. Of special interest are the most powerful AGNs where shocks can accelerate particles to energies well above $\sim 10^{18} \mathrm{eV}$ via the first-order Fermi mechanism [19, 20]. A nearby specially powerful source may be able to reach energies past the cutoff and fit the observed spectrum 
[21]. However, extremely powerful AGNs with radio lobes and hot spots are rare and far apart and are unlikely to match the observed arrival direction distribution. If M87 or CenA are the primary sources of UHECRs, a concentration of events in their directions should be seen at the highest energies. The next known nearby source after M87 is NGC315 which is already too far at a distance of $\sim 80 \mathrm{Mpc}$. Any unknown source between M87 and NGC315 would likely contribute a second hot spot, not the observed isotropic distribution. The very distant radio lobes will contribute a GZK cut spectrum which is not observed yet.

The possibility of stronger Galactic and extragalactic magnetic fields may reduce the problem. In particular, a strong Galactic wind can significantly alter the paths of UHECRs such that the observed arrival directions of events above $10^{20} \mathrm{eV}$ would trace back to the North Galactic Pole which is close to the Virgo cluster where M87 resides [22]. The proposed wind would focus most observed events within a very narrow energy range into the northern Galactic pole and render point source identification fruitless. Full sky coverage of future experiments will be a key discriminator of such proposals.

In Figure 8, the smallest objects of interest are neutron stars. Neutron star not only have the ability to confine $10^{20} \mathrm{eV}$ protons, the rotation energy of young neutron stars is more than sufficient to match the observed UHECR fluxes [23]. However, ambient magnetic and radiation fields induce significant losses inside a neutron star's light cylinder. The plasma that expands beyond the light cylinder is free from the main loss processes and may be accelerated to ultra high energies. In particular, newly formed, rapidly rotating neutron stars may accelerate iron nuclei to UHEs through relativistic MHD winds beyond their light cylinders [24]. This mechanism naturally leads to very hard injection spectra $(\gamma \simeq 1)$. In this case, UHECRs originate mostly in the Galaxy and the arrival directions require that the primaries be heavier nuclei. Depending on the structure of Galactic magnetic fields, the trajectories of iron nuclei from Galactic neutron stars can be consistent with the observed arrival directions of the highest energy events (see, e.g., [25]). This proposal should be constrained once the primary composition is clearly determined.

Transient high energy phenomena such as gamma-ray bursts (GRBs) may also be a source of ultra-high energies protons [26, 27]. In addition to both phenomena having unknown origins, GRBs and UHECRs have other similarities that may argue for a common source. Like UHECRs, GRBs are distributed isotropically in the sky, and the average rate of $\gamma$-ray energy emitted by GRBs is comparable to the energy generation rate of UHECRs of energy $>10^{19} \mathrm{eV}$ in a redshift independent cosmological distribution of sources. However, recent GRB counterpart identifications argue for a strong cosmological evolution for GRBs. The distribution of UHECR arrival directions and arrival times argues against the GRB-UHECR common origin. Events past the GZK cutoff require that only GRBs from $\lesssim 50 \mathrm{Mpc}$ contribute. Since less than about one burst is expected to have occurred within this region over a period of $100 \mathrm{yr}$, the unique source would appear as a concentration of UHECR events in a small part of the sky. In addition, the signal would be very narrow in energy $\Delta E / E \sim 1$. Again, a strong intergalactic magnetic field can ease the arrival direction difficulty dispersing the events of a single burst but also decreasing the flux below the observed level depending on burst characteristics.

\subsection{New Physics Models}

The UHECR puzzle has inspired a number of models that involve physics beyond the stan- 


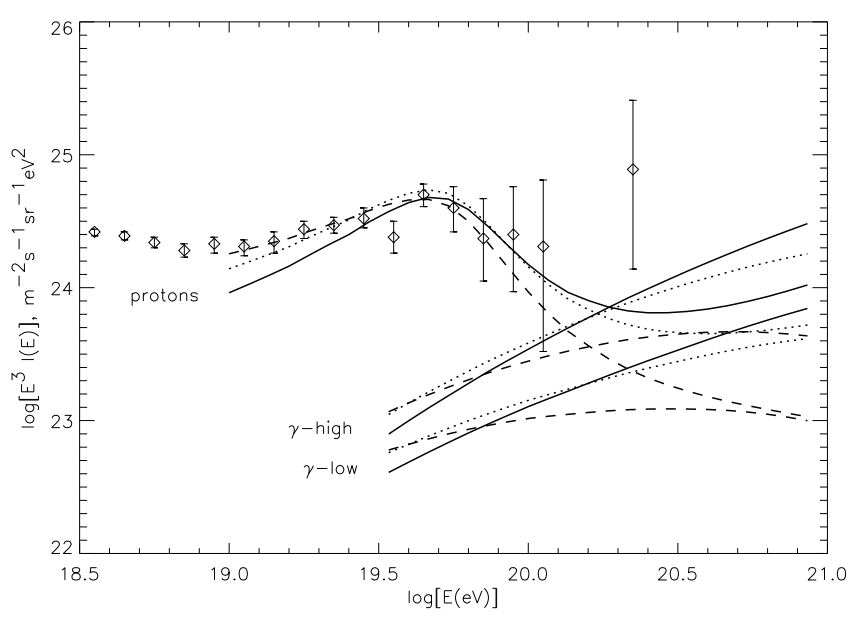

Figure 9: Proton and $\gamma$-ray fluxes from necklaces for $m_{X}=10^{14} \mathrm{GeV}$ (dashed lines), $10^{15} \mathrm{GeV}$ (dotted lines), and $10^{16} \mathrm{GeV}$ (solid lines) normalized to the observed data. $\gamma$-high and $\gamma$-low correspond to two extreme cases of $\gamma$-ray absorption.

dard model of particle physics. New Physics proposals can be top-down models or a hybrid of astrophysical Zevatrons with new particles. Top-down models involve the decay of very high mass relics that could have formed in the early universe.

The most economical among hybrid proposals involves a familiar extension of the standard model, namely, neutrino masses. If some flavor of neutrinos have mass $(\sim 0.1 \mathrm{eV})$, the relic neutrino background is a target for extremely high energy neutrinos to interact and generate other particles through the Z-pole [28, 29]. This proposal requires very luminous sources of extremely high energy neutrinos throughout the universe. Neutrino energies need to be $\gtrsim 10^{21} \mathrm{eV}$ which implies primary protons in the source with energies $\gtrsim 10^{23} \mathrm{eV}$. The decay products of the Z-pole interaction are dominated by photons, which gives a clear test to this proposal. In addition, the neutrino background only clusters on large scales, so the arrival direction for events should be mostly isotropic. Preserving a small scale clustering may be another challenge to this proposal.

If none of the astrophysical scenarios or the hybrid new physics models are able to explain present and future UHECR data, one alternative is to consider top-down models. The idea behind these models is that relics of the very early universe, topological defects (TDs) or superheavy relic (SHR) particles, produced after or at the end of inflation, can decay today and generate UHECRs. Defects, such as cosmic strings, domain walls, and magnetic monopoles, can be generated through the Kibble mechanism as symmetries are broken with the expansion and cooling of the universe. Topologically stable defects can survive to the present and decompose into their constituent fields as they collapse, annihilate, or reach critical current in the case of superconducting cosmic strings $[30,31]$. The decay products, superheavy gauge and higgs bosons, decay into jets of hadrons, mostly pions. Pions in the jets subsequently decay into $\gamma$-rays, electrons, and neutrinos. Only a few percent of the hadrons are expected to be nucleons. Typical features of these scenarios are a predominant release of $\gamma$-rays and neutrinos and a QCD fragmentation spectrum which is considerably harder than the case of Zevatron shock acceleration.

$\mathrm{ZeV}$ energies are not a challenge for top-down models since symmetry breaking scales at the 


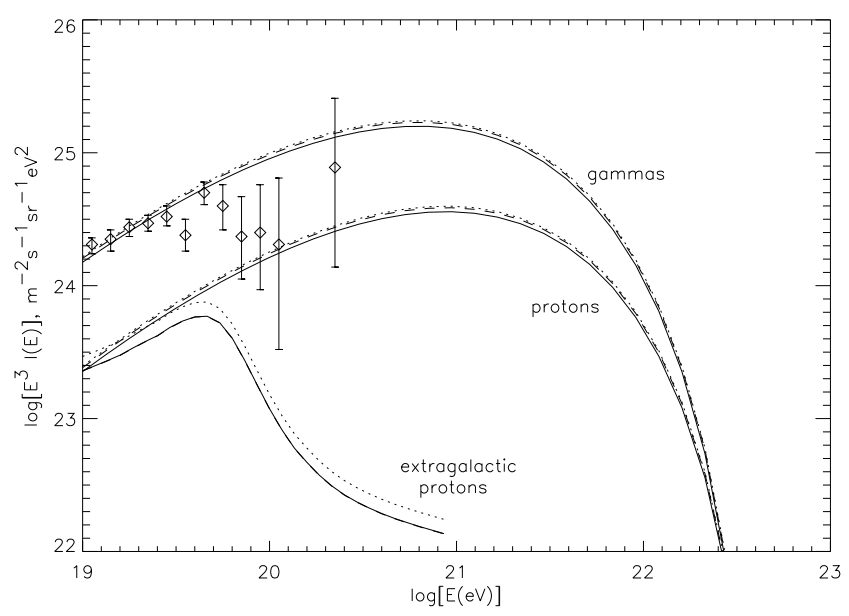

Figure 10: SHRs or monopolia decay fluxes (for $m_{X}=10^{14} \mathrm{GeV}$ ): nucleons from the halo (protons), $\gamma$-rays from the halo (gammas) and extragalactic protons. Solid, dotted and dashed curves correspond to different model parameters.

end of inflation typically are $\gg 10^{21} \mathrm{eV}$. Fitting the observed flux of UHECRs is harder since the typical distances between TDs is the Horizon scale or several Gpc. The low flux hurts proposals based on ordinary and superconducting cosmic strings which are distributed throughout space. Monopoles usually suffer the opposite problem, they would in general be too numerous. Inflation succeeds in diluting the number density of monopoles and makes them too rare for UHECR production. Once two symmetry breaking scales are invoked, a combination of horizon scales gives room to reasonable fluxes. This is the case of cosmic necklaces [32] which are hybrid defects where each monopole is connected to two strings resembling beads on a cosmic string necklace. The UHECR flux which is ultimately generated by the annihilation of monopoles with antimonopoles trapped in the string [33]. In these scenarios, protons dominate the flux in the lower energy side of the GZK cutoff while photons tend to dominate at higher energies depending on the radio background (see Fig. 6). If future data can settle the composition of UHECRs from 0.01 to $1 \mathrm{ZeV}$, these models can be well constrained. In addition to fitting the UHECR flux, topological defect models are constrained by limits from EGRET on the flux of photons from $10 \mathrm{MeV}$ to $100 \mathrm{GeV}$.

Another interesting possibility is the proposal that UHECRs are produced by the decay of unstable superheavy relics that live much longer than the age of the universe [34]. SHRs may be produced at the end of inflation by non-thermal effects such as a varying gravitational field, parametric resonances during preheating, instant preheating, or the decay of topological defects. These models need to invoke special symmetries to insure unusually long lifetimes for SHRs and that a sufficiently small percentage decays today producing UHECRs. As in the topological defects case, the decay of these relics also generates jets of hadrons. These particles behave like cold dark matter and could constitute a fair fraction of the halo of our Galaxy. Therefore, their halo decay products would not be limited by the GZK cutoff allowing for a large flux at UHEs (see Fig. 7). Similar signatures can occur if topological defects are microscopic, such as monopolonia and vortons, and decay in the Halo of our Galaxy. In both cases the composition of the primary would be a good discriminant since the decay products are usually dominated by photons. In the case of 


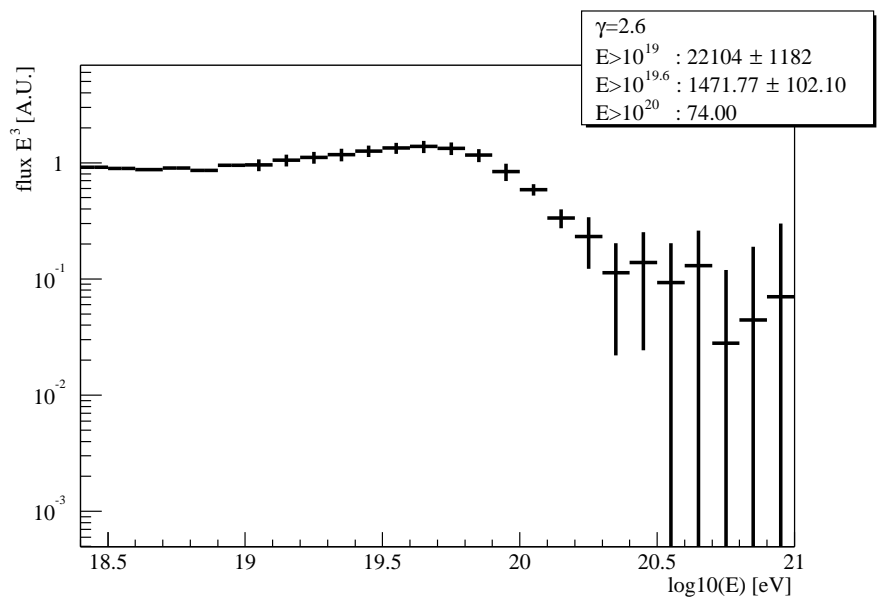

Figure 11: Auger South statistics at the GZK feature.

SHR decays, the arrival direction distribution should be close to isotropic but show an asymmetry due to the position of the Earth in the Galactic Halo [33] and the clustering due to small scale dark matter inhomogeneities [35].

\section{Preview of the Next Generation}

Neither AGASA nor HiRes have the necessary statistics and control of systematics to determine in a definitive way the existence of either the GZK feature or of a novel source of Super-GZK events. Moreover, if the AGASA clusters are an indication of point sources of UHECRs, a large number of events per source will be necessary to study their nature. In order to discover the origin of UHECRs, much larger aperture observatories are now under construction, i.e., the Pierre Auger Project [36], or under development, i.e., the Telescope Array [37], the Extreme Universe Space Observatory [38], and the Orbiting Wide-field Light-collectors (OWL) mission [39].

The Pierre Auger Project will consist of two giant airshower arrays one in the South and one in the North each with 1600 water Cherenkov detectors covering $3000 \mathrm{~km}^{2}$ and four sites of fluorescence telescopes. Auger is being built to determine the spectrum, arrival direction, and composition of UHECR in a full sky survey. The survey should provide large event statistics and control of systematics through detailed detector calibration of the surface array and fluorescence detectors individually in addition to the cross-calibration of the two detection techniques through the observation of hybrid and stereo-hybrid events. Depending on the UHECR spectrum, Auger should measure the energy, direction and composition of about 60 events per year above $10^{20} \mathrm{eV}$ and about 6000 events per year above $10^{19} \mathrm{eV}$ (see Figure 11). In addition, it should be able to detect a few neutrino events per year if the UHECRs are extragalactic protons.

The Auger surface array is composed of stand alone 1.5 meter tall water tanks that are powered by solar cells, timed by GPS systems, and communicate via radio antennas. Three photomultipliers per tank register the Cherenkov light when shower particles cross the tanks. Having three photomultipliers per tank allows the self-calibration of each tank in the field. The height of the tanks makes the ground array an excellent detector for inclined showers. Inclined showers and their 
asymmetries allow for a novel method for composition studies and for the detection of neutrino showers from horizontal and Earth skimming high energy neutrinos.

The fluorescence detectors at the Auger observatory have a complete calibration system. The atmospheric monitoring includes lasers, lidars, ballon radio sondes, cloud monitors, and movable calibration light sources [40]. In addition, the whole telescopes including mirrors are calibrated from front to end with light sources. Hybrid detection is a powerful measurement of individual showers and can be used to reach large statistics on energies down to $10^{18} \mathrm{eV}$ with the use of fluorescence and a small number of tanks per event. The ability to study events at $10^{18} \mathrm{eV}$ in the Southern hemisphere will be crucial in confirming the reported anisotropies toward the Galactic Center region. The combination of mono fluorescence events that trigger even a single tank allows for great angular reconstruction of events comparable to stereo events.

The Auger collaboration consists of about 250 scientists from 16 countries. The Southern Auger Observatory is already operating with 500 surface detector tanks deployed and two fluorescence telescope sites completed. The first science results of the observatory should be presented in the Summer of 2005.

Another upcoming experiment is the recently approved Telescope Array (TA) which consists of a hybrid detector of three fluorescence telescopes overlooking a scintillator array. The array would cover about $400 \mathrm{~km}^{2}$ with $1.2 \mathrm{~km}$ spacing. The design limits the exposure at theh ighest energies but is suited to energies from $\sim 10^{17} \mathrm{eV}$ to $\sim 10^{20} \mathrm{eV}$, where a transition between Galactic and extragalactic UHECRs are expected. TA should be able to see some super-GZK events but with significantly smaller statistics than the Auger project. Instead, TA is planning to concentrate their efforts in having a broad reach in energies to study the spectrum and composition through the transition from Galactic to extragalactic that may involve a simultaneous heavy to light primaries transition.

Finally, the Extreme Universe Space Observatory (EUSO) is a fluorescence detector designed for the International Space Station (ISS) aiming at observations of extremely high energy cosmicrays (EHECRs), i.e., cosmic rays between $10^{20}$ and $10^{22} \mathrm{eV}$. EUSO will observe showers from above the atmosphere and will have full sky coverage due to the ISS orbit. This project is a good complement to ground arrays since, it will focus on larger energy scales and will have different systematic effects. Their threshold may be above $5 \times 10^{19} \mathrm{eV}$ depending on technical features of the fluorescence detectors. The telescope's expected angular resolution is $\sim 0.2$ degrees and the energy resolution about $\sim 20 \%$. The aperture may reach $3 \times 10^{6} \mathrm{~km}^{2}$-sterad with a $10 \%$ duty cycle. This can translate into about 3000 events per year for energies above $10^{20} \mathrm{eV}$ (see Fig. 12).

On an even larger scale, the future OWL mission will consist of a pair of satellites placed in tandem in a low inclination, medium altitude orbit. The OWL telescopes will point down at the Earth and will together point at a section of atmosphere about $\sim 6 \times 10^{5} \mathrm{~km}^{2}$. The large aperture should translate to high statistics at the highest energies and the stereo capabilities of the two satellite design will help control systematics at the largest energies.

\section{Conclusion}

After decades of attempts to discover the origin of ultra-high energy cosmic rays, present results are still inconclusive. The results from past experiments show the need to understand and 


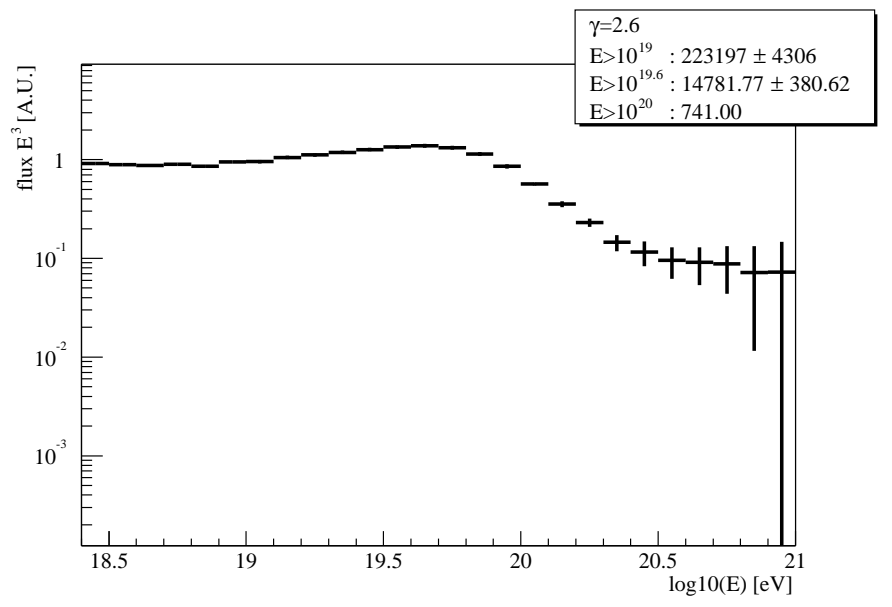

Figure 12: EUSO statistics at the GZK feature.

control systematic effects within each technique and to cross-calibrate the two techniques presently available for UHECR studies (ground arrays and fluorescence). In addition, the lack of sufficient statistics limits the discussion of an excess flux or a drop in flux around the GZK feature. Next generation experiments are gearing up to accumulate the necessary statistics while having a better handle on the systematics. In the following decade, we may see the growth of a new astronomy with ultra-high energy charged particles and finally resolve the almost century old puzzle of the origin of cosmic rays.

\section{Acknowledgements}

Thanks to the organizers of the 28th Johns Hopkins Workshop on Current Problems in Particle Theory for a great meeting. This work was supported in part by the KICP under NSF PHY-0114422 , by the NSF through grant AST-0071235, and the DOE grant DE-FG0291-ER40606.

\section{References}

[1] P. Auger, et al., Comptes Rendus 206, 1721 (1938); Rev. Mod. Phys. 11, 288 (1939).

[2] K.-H. Kampert, et al, (KASCADE-Grande Collaboration), Acta Phys.Polon. B35 1799 (2004).

[3] R.U. Abbasi et al. (HiRes Collaboration), Ap. J. submitted (2004); arXiv:astro-ph/0407622

[4] K. Greisen, Phys. Rev. Lett. 16, 748 (1966); G. T. Zatsepin and V. A. Kuzmin, Sov. Phys. JETP Lett. 4, 78 (1966).

[5] P. Bhattacharjee and G., Sigl, Phys. Rept. 327, 109 (2000)

[6] A. V. Olinto, Phys. Rep. 333, 329 (2000); J. W. Cronin, Proceedings of TAUP 2003, astro-ph/0402487, F.W. Stecker, J.Phys. G 29 R47(2003).

[7] M. Takeda, et al. (AGASA Collaboration), in the Proceedings of the 28th International Cosmic Ray Conference, Tsukuba, Japan, (2003).

[8] M. Takeda, et al. (AGASA Collaboration), Ap. J. 522, 225 (1999). 
[9] R.U. Abbasi, et al. (HiRes Collaboration), Phys.Rev.Lett. 92, 151101(2004).

[10] P. P. Kronberg, Rep. Prog. Phys., 57, 325 (1994).

[11] P. Blasi, S. Burles, and A. V. Olinto, Ap. J. Lett. 514 L79 (1999).

[12] K. Dolag, D. Grasso, V. Springel, and I. Tkachev, submitted JCAP (2004), arXiv:astro-ph/0410419

[13] R.U. Abbasi, et al. (HiRes Collaboration), Astropart. Phys. 22, 139 (2004); Ap.J. 610, L73 (2004).

[14] M. Takeda, et al (AGASA Collaboration), Astropart.Phys. 19, 447 (2003).

[15] D. DeMarco , P. Blasi, and A. V. Olinto, Astropart.Phys. 20, 53 (2003).

[16] D. Bergman, et al., (HiRes Collaboration) in the Proceedings of the 28th International Cosmic Ray Conference, Tsukuba, Japan, (2003).

[17] A.M. Hillas, Ann. Rev. Astron. Astrop. 22425 (1984).

[18] H. Kang, D. Ryu, and T.W. Jones, Astropart. Phys. 456422 (1996).

[19] P.L. Biermann and P. Strittmatter, Astropart. Phys., 322643 (1987).

[20] J.P. Rachen and P.L. Biermann, Astron. and Astrop. 272 (1993) 161.

[21] P. Blasi and A.V. Olinto, Phys. Rev. D 59 (1999) 023001.

[22] E.J. Ahn, P.L. Biermann, G. Medina-Tanco, and T. Stanev, astro-ph/9911123 (1999).

[23] A. Venkatesan, M.C. Miller, and A.V. Olinto, ApJ 484323 (1997).

[24] P. Blasi, R.I. Epstein, and A.V. Olinto, ApJ. Lett. 533 L123 (2000) .

[25] S. O'Neal, P. Blasi, and A.V. Olinto, in the Proceedings of the 27th International Cosmic Ray Conference, Hamburg, Germany (2001).

[26] E. Waxman, Phys. Rev. Lett. 75386 (1995) .

[27] M. Vietri, ApJ 453883 (1995).

[28] D. Fargion, B. Mele, and A. Salis, ApJ, 517725 (1999).

[29] T. Weiler, Astropar. Phys. 11303 (1999).

[30] C.T. Hill, Nucl. Phys. B 22469 (1983).

[31] D.N. Schramm, and C.T. Hill, Proc. 18th ICRC (Bangalore, India) 2393 (1983).

[32] V. Berezinsky and A. Vilenkin, Phys. Rev. Letters, 795202 (1997).

[33] V. Berezinsky, P. Blasi and A. Vilenkin, Phys. Rev. D 58 103515-1 (1998).

[34] V. Berezinsky, M. Kachelrieß and A. Vilenkin, Phys. Rev. Letters 794302 (1997) .

[35] P. Blasi and R. K. Seth, Phys. Lett. B 486233 (2000).

[36] J. W. Cronin, AIP Conf. Proc. 566, 1 (2001).

[37] M. Fukushima, et al. (TA Collaboration) in the Proceedings of the 28th International Cosmic Ray Conference, Tsukuba, Japan, (2003).

[38] M. Teshima, et al. (EUSO Collaboration), in the Proceedings of the 28th International Cosmic Ray Conference, Tsukuba, Japan, (2003).

[39] F.W. Stecker, et al., in the proceedings of CRIS 2004, to be published in Nucl. Phys. B. (2004).

[40] M. D. Roberts, et al. (Auger Collaboration), proceedings of the 28th International Cosmic Ray Conference, Tsukuba, Japan, (2003), arXiv:astro-ph/0308410. $\hat{E}$ 